\title{
The Conservation Laws and Stability of Fluid Waves of Permanent Form
}

\author{
Troyan A. Bodnar' \\ Department of Mathematics Technological Institute, Altai State Technical University, Biisk, Russia \\ Email: bta@bti.secna.ru
}

Received December 13, 2012; revised January 15, 2013; accepted January 23, 2013

\begin{abstract}
The solution of Nekrasov's integral equation is described. By means of this solution the wave kinetic, potential, and full mechanical energies are defined as functions of fluid depth and wavelength.The wave obey the laws of mass and energy conservation. It is found that for any constant depth of fluid the wavelength is bounded from above by a value denoted as maximal wavelength. At maximal wavelength 1) the maximum slope of the free surface of the wave exceeds $38^{\circ}$ and the value $45^{\circ}$ is supposed attainable, 2) the wave kinetic energy vanishes. The stability of a steady wave considered as a compound pendulum is analyzed.
\end{abstract}

Keywords: Nekrasov Integral Equation; Kellogg Method; Successive Approximations Method; Wave Mechanical Energy; Wave Stability

\section{Introduction}

This paper is based on Nekrasov's integral equation solution obtained in $[1,2]$. This solution allows us to find the profile and velocity of a gravitational wave, and the calculation of the wave kinetic and potential energy is possible. At fixed depth of fluid the solution of Nekrasov's integral equation exists on a limited segment of wavelength $\lambda \in\left[0, \lambda_{\max }\right]$. The potential and full mechanical energies are monotonically increasing functions on the segment $\left[0, \lambda_{\max }\right]$. From the law of the change of wave's kinetic energy presented in [3] as mathematical theorem follows that the kinetic energy vanishes on the boundaries of the segment $\left[0, \lambda_{\max }\right]$ and has the maximum at a point $\lambda_{T} \in\left[0, \lambda_{\max }\right]$. Thus we observe symmetry: at the points $\lambda=0, \lambda=\lambda_{\max }$ the full mechanical energy consists only of potential energy. The wave of constant shape may be considered as a compound pendulum with a suspension center in the origin of coordinates arranged on unperturbed surface of fluid.Then the wave stability is spotted as a compound pendulum stability.

The plan of the paper is as follows. In Section 2, we describe the method of Nekrasov's integral equation solution. Here this method has been used for evaluation of the maximum wavelength $\lambda_{\max }$ boundaries and for estimation of the maximum slope of the wave free surface.

In Section 3, geometrical and energy properties of a wave are explored and the theorem about the change of the wave velocity on the segment $\left[0, \lambda_{\max }\right]$ is proved.
As a result we have gained the laws of the change of wave's kinetic and potential energy. Here we have defined the wave's center of mass as a function of depth-towavelength ratio and have made some suppositions concerning the wave stability considering it as a compound pendulum.

\section{Solution of Nekrasov's Integral Equation}

Nekrasov's integral equation describing steady state waves of unchangeable shape on the surface of a fluid with finite depth $h$ is written as [4]

$$
\omega(\theta)=\int_{-\pi}^{\pi} K\left(\theta, \sigma, h_{\lambda}\right) \frac{\mu\left(h_{\lambda}\right) \sin \omega(\sigma)}{1+\mu\left(h_{\lambda}\right) \int_{0}^{\sigma} \sin \omega(s) \mathrm{d} s} \mathrm{~d} \sigma,
$$

where $\theta$ - is the polar angle, $\omega(\theta)$ - is the angle that the wave surface makes with the horizontal, $\lambda$ - is the wavelength, $h_{\lambda}=h \lambda^{-1}, \mu\left(h_{\lambda}\right)$ - is an arbitrary constant,

$$
K\left(\theta, \sigma, h_{\lambda}\right)=\frac{1}{3 \pi} \sum_{k=1}^{\infty} \tanh \left(2 k \pi h_{\lambda}\right) \frac{\sin k \theta \sin k \sigma}{k} .
$$

From Equation (1.1) follows that required function $\omega(\theta)$ represents a trigonometric series

$$
\omega(\theta)=\sum_{k=1}^{\infty} a_{k} \sin k \theta .
$$

The evaluation of scalar product

$$
\langle\omega(\theta), \sin k \theta\rangle=\int_{-\pi}^{\pi} \omega(\theta) \sin k \theta \mathrm{d} \theta, k=1,2, \cdots, n
$$


taking account of Equations (1.1) and (1.2) gives a system of $n$ nonlinear integral equations

$$
a_{k}=\int_{-\pi}^{\pi} \frac{\tanh \left(2 k \pi h_{\lambda}\right) \mu_{n}\left(h_{\lambda}\right) \sin k \sigma \sin \omega_{n}(\sigma)}{3 k \pi\left(1+\mu_{n}\left(h_{\lambda}\right) \int_{0}^{\sigma} \sin \omega_{n}(s) d s\right)} \mathrm{d} \sigma
$$$$
k=1,2, \cdots, n,
$$

where the subscript $n$ denotes that in Equation (1.1) a truncated kernel $K_{n}\left(\theta, \sigma, h_{\lambda}\right)$ with $n$ instead of $\infty$ is used. Below the subscript $n$ of any function will be omitted if

$$
\max _{-\pi \leq \theta \leq \pi}\left|\omega_{n}(\theta)-\omega_{n-1}(\theta)\right|<\varepsilon,
$$

where $\varepsilon$ - is a suitable from accuracy point of view small number

The system (1.3) containing $n+1$ unknowns $a_{1}, a_{2}, \cdots, a_{n}, \mu_{n}\left(h_{\lambda}\right)$ is underdetermined and has a set of solutions including the trivial.

We assume that the first coefficient $a_{1}$ is independent of $h_{\lambda}, \mu_{n}\left(h_{\lambda}\right)$, and $n$ and can be calculated from the linearized on $\mu_{n}\left(h_{\lambda}\right)$ system (1.3) at $n=1$. Thus $a_{1}=a_{1}^{0}, \mu_{1}\left(h_{\lambda}\right)=\mu_{1}^{0}\left(h_{\lambda}\right)$ satisfy the equation

$$
a_{1}^{0}=\frac{\mu_{1}^{0}\left(h_{\lambda}\right)}{3 \pi} \int_{-\pi}^{\pi} \frac{\tanh \left(2 \pi h_{\lambda}\right) \sin \sigma \sin \omega_{1}(\sigma)}{\left(1+\int_{0}^{2 \pi} \sin \omega_{1}(s) \mathrm{d} s\right)} \mathrm{d} \sigma
$$

This equation has been solved by Kellogg's method [5] in $[1,2]$. Let us remark that the Kellogg method was applied to a non-linear integral equation and the discovered solution is not spectral. In accordance with [2], Equation (1.5) has a unique solution (the motionless point) $a_{1}^{0}=0.0474520, \mu_{1}^{0}\left(h_{\lambda}\right)=\pi \operatorname{coth}\left(2 \pi h_{\lambda}\right)$.

Now we fix in (1.3) $a_{1}=a_{1}^{0}$ and consider $\mu_{1}^{0}\left(h_{\lambda}\right)$ as the initial approximation of $\mu_{n}\left(h_{\lambda}\right)$. After that, the system of $n$ Equations (1.3) contains $n$ unknowns $a_{2}, a_{3}, \cdots, a_{n}, \mu_{n}\left(h_{\lambda}\right)$ and has a solution that cannot be trivial.It has been shown [1,2] that coefficient $a_{1}^{0}$ satisfy system (1.3) at $n=1$ if

$h_{\lambda} \in\left[h_{\lambda 1}^{*}, \infty\right], \mu_{1}\left(h_{\lambda}\right) \in[3.47841, \infty]$, where

$h_{\lambda 1}^{*}=0.0113482$ (in [2] $h_{\lambda 1}^{*}$ is designated as $h_{\lambda}^{*}$ ). This means that the solution of the system (1.3) at $n=1$ exists and is unique if the wavelength $\lambda \in\left[0, \lambda_{1 \max }\right]$, where $\lambda_{\max }=h\left(h_{\lambda 1}^{*}\right)^{-1}$.

The lower boundary of the segment $\left[h_{\lambda n}^{*}, \infty\right]$ on which coefficient $a_{1}^{0}$ satisfy system (1.3) at $n>1$ can be obtained by solving the system

$$
a_{k}=\int_{-\pi}^{\pi} \frac{\tanh \left(2 k \pi h_{\lambda n}^{*}\right) \sin k \sigma \sin \omega_{n}(\sigma)}{3 k \pi \int_{0}^{\sigma} \sin \omega_{n}(s) \mathrm{d} s} \mathrm{~d} \sigma, k=1,2, \cdots, n,
$$

derived from (1.3) by substitution $\mu_{n}\left(h_{\lambda}\right)=\infty$. If re- quirements (1.4) are satisfied, then the function $\omega(\theta)$ is defined on the segment $\mu\left(h_{\lambda}\right) \in[3.48971 ; \infty]$ (see [1]), or on the equivalent segment

$$
h_{\lambda} \in\left[h_{\lambda}^{*}, \infty\right], \mu\left(h_{\lambda}^{*}\right)=\infty, \mu(\infty)=3.48971 .
$$

The system (1.6) containing $n$ unknowns $a_{2}, a_{3}, \cdots, a_{n}, h_{\lambda n}^{*}$ was solved by successive approximations method at $n=10$ limited by computer's throughput. The results of calculations of $h_{\lambda 10}^{*}, a_{1}, a_{2}, \cdots, a_{10}$ are given below:

$$
\begin{aligned}
& h_{\lambda 10}^{*}=0.0482209, a_{1}=0.0474520, a_{2}=0.0811896, \\
& a_{3}=0.0982136, a_{4}=0.103116, a_{5}=0.101298, \\
& a_{6}=0.0963571, a_{7}=0.0902088, a_{8}=0.0837567, \\
& a_{9}=0.0774002, a_{10}=0.0713327 .
\end{aligned}
$$

The function $\omega_{10}(\theta)$ is of low accuracy at the point $\left(h_{\lambda 10}^{*}, \mu_{10}\right)=(0.0482209, \infty)$ and allows only to write the inequalities $h_{\lambda 10}^{*}<h_{\lambda}^{*}<0.06$. From these inequalities follows that the maximal wavelength $\lambda_{\max }=h\left(h_{\lambda}^{*}\right)^{-1}$ is bounded on both sides $16.666667 h<\lambda_{\max }<20.737896 h$. The results of calculations of $\mu_{10}(0.06), a_{1}, a_{2}, \cdots, a_{10}$ for $h_{\lambda}=0.06$ are given below:

$$
\begin{aligned}
& \mu_{10}(0.06)=30.575149, a_{1}=0.0474520, a_{2}=0.0635455, \\
& a_{3}=0.0584506, a_{4}=0.0475204, a_{5}=0.0370809, \\
& a_{6}=0.0286028, a_{7}=0.0220196, a_{8}=0.0169372, \\
& a_{9}=0.0129768, a_{10}=0.00985512 .
\end{aligned}
$$

These numerical results assert that $a_{1}^{0}$ will remain the solution of system (1.3) at $h_{\lambda}=0.06$ for any $n>10$.

From the solution of the system (1.6) we obtain the coefficients of the function $\omega_{10}(\theta)$. This function achieves the maximum $\left|\omega_{10}^{\max }\left( \pm \theta_{10}^{0}\right)\right|=0.670527$ at the points $\theta_{10}^{0}= \pm 0.229065$. The maximum slope of the free surface in degrees $\left|\omega_{10}^{\max }\left( \pm \theta_{10}^{0}\right)\right|=38.418367^{\circ}$ exceeds the value $30.3787032466^{\circ}$ received in [6]. We suppose that $\left|\omega_{10}^{\max }\left( \pm \theta_{10}^{0}\right)\right|<\left|\omega^{\max }\left( \pm \theta^{0}\right)\right| \leq 0.25 \pi, \theta^{0}<\theta_{10}^{0}$.

\section{The Conservation Law of Full Mechanical Energy and Stability of a Wave}

For evaluation of a wave square and full mechanical energy we need the parametric equations of wave's surface coordinates

$$
\begin{aligned}
& x(\theta)=-\frac{\lambda}{2 \pi}\left(\theta+b_{1} \sin \theta+\frac{1}{2} b_{2} \sin 2 \theta+\frac{1}{3} b_{3} \sin 3 \theta+\cdots\right), \\
& y(\theta)=\frac{\lambda}{2 \pi}\left(b_{1} \cos \theta+\frac{1}{2} b_{2} \cos 2 \theta+\frac{1}{3} b_{3} \cos 3 \theta+\cdots\right),
\end{aligned}
$$


and the function

$$
R(\theta)=\exp \left(a_{1} \cos \theta+a_{2} \cos 2 \theta+a_{3} \cos 3 \theta+\cdots\right) .
$$

Coefficients $b_{1}, b_{2}, \cdots$ are determined as algebraic expressions of $a_{1}, a_{2}, \cdots$ [2].

The coordinate origin $O$ is on vertical line through the wave crest at distance $h$ from the bottom,the $O y$ axis is directed upward, and the $O x$ axis to the right. In a coordinate system attached to the wave the bottom moves from right to left at velocity [4]

$$
c(\lambda)=\left[\frac{3 g \lambda R^{3}(0)}{2 \pi \mu\left(h_{\lambda}\right)}\right]^{\frac{1}{2}} .
$$

Using (2.1)-(2.3) at fixed $h_{\lambda}$, we obtain the expressions for the properties of the wave: the surface area

$$
S_{w}=\int_{-\pi}^{\pi} x(\theta) y^{\prime}(\theta) \mathrm{d} \theta ;
$$

the coordinates of the center of mass

$$
x_{c}=0, y_{c}=\frac{1}{S_{w}} \int_{-\pi}^{\pi} x(\theta) y(\theta) y^{\prime}(\theta) \mathrm{d} \theta ;
$$

the kinetic energy

$$
T_{w}=\int_{-\pi}^{\pi} \frac{\rho c^{2}(\lambda) x(\theta) y^{\prime}(\theta) \mathrm{d} \theta}{2 R^{2}(\theta)},
$$

where constant $\rho$ denotes the fluid density; the potential energy

$$
\Pi_{w}=g \rho S_{w}\left(h+y_{c}\right) ;
$$

the full mechanical energy

$$
E_{w}=T_{w}+\Pi_{w} .
$$

The full mechanical energy of an unperturbed fluid layer with depth $h$ and wavelengths $\lambda$ is considered as

$$
\Pi_{0}=0.5 g \rho h^{2} \lambda .
$$

Substituting $h h_{\lambda}^{-1}$ for $\lambda$ in (2.4)-(2.9), we get

$$
\begin{aligned}
& S_{w}\left(h_{\lambda}\right)=h^{2} S_{w}^{1}\left(h_{\lambda}\right), y_{c}\left(h_{\lambda}\right)=h y_{c}^{1}\left(h_{\lambda}\right), \\
& T_{w}\left(h_{\lambda}\right)=g \rho h^{3} T_{w}^{1}\left(h_{\lambda}\right), \\
& \Pi_{w}\left(h_{\lambda}\right)=g \rho h^{3}\left(1+y_{c}^{1}\left(h_{\lambda}\right)\right) S_{w}^{1}\left(h_{\lambda}\right),
\end{aligned}
$$

and $\Pi_{0}\left(h_{\lambda}\right)=0.5 g \rho h^{3} h_{\lambda}^{-1}$, where

$$
\begin{aligned}
& S_{w}^{1}\left(h_{\lambda}\right)=S_{w}(h=1), y_{c}^{1}\left(h_{\lambda}\right)=y_{c}(h=1), \\
& T_{w}^{1}\left(h_{\lambda}\right)=(g \rho)^{-1} T_{w}(h=1)
\end{aligned}
$$

are integrals depending on parameter $h_{\lambda}$. Using these relationships we can write the Equations (2.6)-(2.8) in the form

$$
\begin{aligned}
& T_{w}\left(h_{\lambda}\right)=C_{T}\left(h_{\lambda}\right) \Pi_{0}, \Pi_{w}\left(h_{\lambda}\right)=C_{\Pi}\left(h_{\lambda}\right) \Pi_{0}, \\
& E_{w}\left(h_{\lambda}\right)=C_{E}\left(h_{\lambda}\right) \Pi_{0},
\end{aligned}
$$

where

$$
\begin{aligned}
& C_{T}\left(h_{\lambda}\right)=2 h_{\lambda} T_{w}^{1}\left(h_{\lambda}\right), C_{\Pi}\left(h_{\lambda}\right)=2 h_{\lambda}\left(1+y_{c}^{1}\left(h_{\lambda}\right)\right) S_{w}^{1}\left(h_{\lambda}\right) \\
& C_{E}\left(h_{\lambda}\right)=C_{T}\left(h_{\lambda}\right)+C_{\Pi}\left(h_{\lambda}\right) .
\end{aligned}
$$

The solution of system (1.3) obtained in [2] allow us to calculate the function $C_{T}\left(h_{\lambda}\right), C_{\Pi}\left(h_{\lambda}\right), C_{E}\left(h_{\lambda}\right)$ on the segment $[0.08,1.4]$. On the boundaries of this segment we have

$$
\begin{aligned}
& C_{T}(0.08)=0.0585045, C_{T}(1.4)=0.000615295, \\
& C_{\Pi}(0.08)=0.202921, C_{\Pi}(1.4)=0.0105552, \\
& C_{E}(0.08)=0.261226, C_{E}(1.4)=0.0111705 .
\end{aligned}
$$

The functions $C_{T}\left(h_{\lambda}\right), C_{\Pi}\left(h_{\lambda}\right), C_{E}\left(h_{\lambda}\right)$ calculated on the segment $[0.08 ; 0.4]$ are shown on Figure 1. The outcomes of these calculations allow to assert that the wave potential energy $\Pi_{w}\left(h_{\lambda}\right)$ as function of parameter $h_{\lambda}$ monotonically diminishes on the segment $\left[h_{\lambda}^{*}, \infty\right]$.

In order to calculate the greatest value of potential energy of a wave we need the $h_{\lambda}^{*}$ solution of system (1.6) instead of $h_{\lambda 10}^{*}$ (see above).

The law of the wave kinetic energy change is formulated in the form of a mathematical theorem in [3]. This theorem asserts that: 1) for any constant depth of fluid $h$ the wavelength $\left.\lambda \in\left[0, \lambda_{\max }\right] ; 2\right)$ at the boundaries of the segment $\left[0, \lambda_{\max }\right]$ the wave velocity $\left.c(0)=c\left(\lambda_{\max }\right)=0 ; 3\right)$ there is a value $\lambda_{0}$, at which $\mathrm{d} c\left(\lambda_{0}\right) / \mathrm{d} \lambda=0$, and the wave velocity is maximum, i.e. $c\left(\lambda_{0}\right)=c_{\max }$.

Let's prove the points 1) - 3). 1) From (1.7) follows that $h_{\lambda}^{*} \leq h_{\lambda} \leq \infty$ and therefore $0 \leq \lambda \leq h\left(h_{\lambda}^{*}\right)^{-1}=\lambda_{\max }$. 2) Suppose the solution $h_{\lambda}^{*}, \omega(\sigma)$ of (1.6) is known and satisfy (1.4). Using this solution we write the integrals

$$
\begin{aligned}
& a_{k}^{*}=\int_{-\pi}^{\pi} \frac{\tanh \left(2 \pi h_{\lambda}^{*}\right) \sin k \sigma \sin \omega(\sigma)}{3 \pi \int_{0}^{\sigma} \sin \omega(s) \mathrm{d} s} \mathrm{~d} \sigma, \\
& k=1,2, \cdots .
\end{aligned}
$$

The first integral is known $a_{1}^{*}=a_{1}^{0}$; the integral $a_{k}^{*}$ tends to zero as $k \rightarrow \infty$ (see [7]). Now it is possible to express the coefficients $a_{k}, k=1,2, \cdots$ from the system (1.6) in terms of the integrals $a_{k}^{*}, k=1,2, \cdots$. We have

$$
a_{k}=\frac{\tanh \left(2 \pi k h_{\lambda}^{*}\right)}{\tanh \left(2 \pi h_{\lambda}^{*}\right)} \frac{a_{k}^{*}}{k} .
$$

Taking into account $a_{k}^{*} \rightarrow 0$ as $k \rightarrow \infty$, from (2.12) follows that series $\ln R(0)=a_{1}+a_{2}+\cdots$ is converging independently of convergence or divergence of the series $a_{1}^{*}+a_{2}^{*}+\cdots$. Now if we recall (1.7), (2.3), and (2.12), we get $c\left(\lambda_{\max }\right)=0$. 


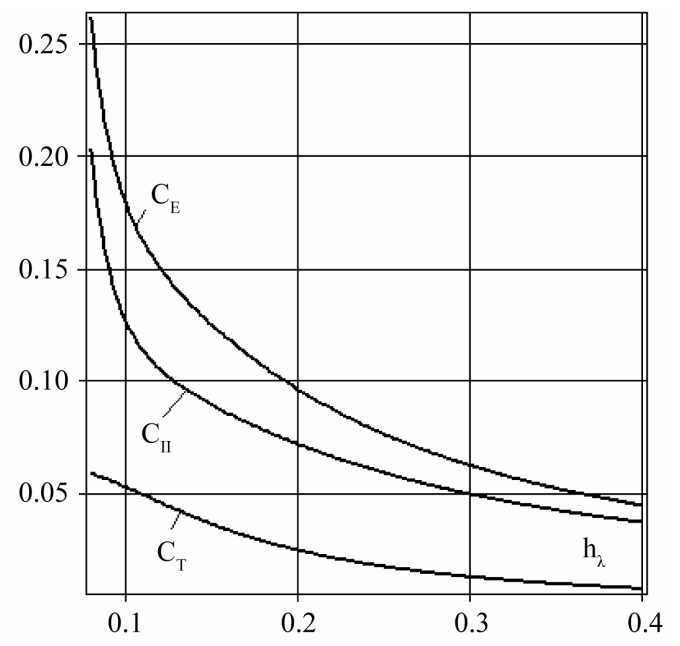

Figure 1. The functions $C_{T}\left(h_{\lambda}\right), C_{\mathrm{II}}\left(h_{\lambda}\right), C_{E}\left(h_{\lambda}\right)$ for $0.08 \leq h_{\lambda} \leq$ 0.4 .

Let as remark that the convergence of series $\omega(\theta)$ has been proved at a limited $\mu$ in [4] and for a $\mu$ presented as a converging series in [8].

3) Since the function $c(\lambda)$ vanishes at the boundaries of the interval $\left[0, \lambda_{\max }\right]$, it follows from the Rolles theorem [7] that exists at least a point

$\lambda_{0} \in\left[0, \lambda_{\max }\right]$ such that $\frac{\mathrm{d} c\left(\lambda_{0}\right)}{\mathrm{d} \lambda}=0$. As the numerator and denominator of right side of Equation (2.3) are strictly increasing functions of $\lambda$ the point $\lambda_{0}$ is single and $c\left(\lambda_{0}\right)=c_{\max }$. The values

$\lambda_{0}=10.791072 h, c_{\max }=0.897495 \sqrt{g h}$ have been calculated in [2].

The kinetic energy of the wave vanishes at boundaries of the segment $\left[0, \lambda_{\max }\right]$ and reaches the maximum at a point $\lambda_{T}>\lambda_{0}$. (This point is not presented on Figure 1). The full mechanical energy of a wave is a monotonically decreasing function on the segment $\left[h_{\lambda}^{*}, \infty\right]$, but vanishes only at the point $\lambda=0$. We note also that the profiles of the waves calculated on the segment $[0.08,1.4]$ obey the law of mass conservation $S_{w}\left(h_{\lambda}\right)=-y(\pi) h h_{\lambda}^{-1}$.

The stability of Nekrasov's waves has been considered in [9]. We proceed from the fact that a liquid maintaining the invariable shape without a vessel, does not suspect that is a fluid.We suppose that a steady state wave can be presented as a compound pendulum with a suspension center in the origin of coordinates. This wave is stable if $y_{c}\left(h_{\lambda}\right)<0$ and unstable if $y_{c}\left(h_{\lambda}\right)>0$. The function

$y_{c}\left(h_{\lambda}\right)$ is presented on Figure 2. This function has the greatest value $y_{c}\left(h_{\lambda}^{*}\right)$, is equal to zero

$y_{c}(0.0851417)=0$, has the minimum

$y_{c}(0.133136)=-0.00889279 h$, and monotonically increases on the interval $[0.133136, \infty]$ reaching the value $y_{c}=0$ only at the point $\lambda=0$. If we continue to con-

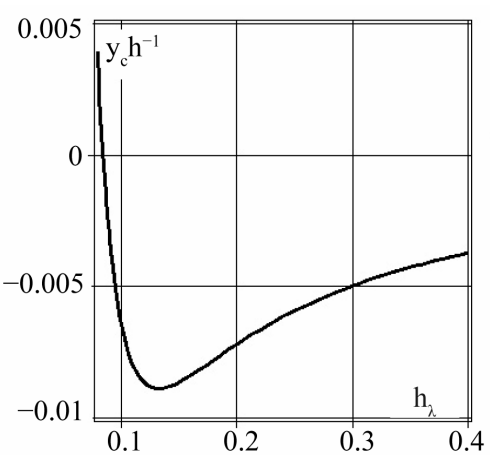

Figure 2. The dependence $y_{c} h^{-1}\left(h_{\lambda}\right)$ for $0.08 \leq h_{\lambda} \leq 0.4$.

sider the wave behaving as a compound pendulum then it is stable at $h_{\lambda} \in(0.0851417, \infty)$ and unstable when

$h_{\lambda} \in\left(h_{\lambda}^{*}, 0.0851417\right)$.

In particular let's consider a wave of length $\lambda=1000 \mathrm{~m}$ on the surface of a fluid with depth $h=100 \mathrm{~m}$ and density $\rho=1000 \mathrm{~kg} / \mathrm{m}^{2}$. The solution of system (1.3) at the point $h_{\lambda}=0.1$ (see [2]) and the coefficients $b_{1}$, $b_{2}, \cdots, b_{7}$ are given below: $\mu_{7}(0.1)=7.449619 ; a_{1}=$ $0.047452 ; a_{2}=0.0195554 ; a_{3}=0.00606262 ; a_{4}=$ $0.00183319 ; a_{5}=0.000565461 ; a_{6}=0.000178475 ; a_{7}=$ $0.0000568810 ; b_{1}=0.047452 ; b_{2}=0.0206812 ; b_{3}=$ $0.00700837 ; b_{4}=0.00233431 ; b_{5}=0.000787255 ; b_{6}=$ $0.000268797 ; b_{7}=0.0000921207$. Using these outcomes in Formulas (2.1)-(2.10) we get the parameters of the wave: the crest coordinate $y(0) \approx 9.7 \mathrm{~m}$; the trough coordinate $y(\pi) \approx-6.2 \mathrm{~m}$; the amplitude $H \approx 15.9 \mathrm{~m}$; the coordinates of mass center $x_{c}=0, y_{c} \approx-0.6 \mathrm{~m}$; the kinetic energy $T_{w} \approx 2.6 \times 10^{6} \mathrm{~kJ}$; the potential energy $\Pi_{w} \approx 6.2 \times 10^{6} \mathrm{~kJ} ;$ the velocity $c \approx 28.1 \mathrm{~m} / \mathrm{s}$. This wave similar to a tsunami is stable as $y_{c}(0.1)<0$.

For $h=100 \mathrm{~m}$ and $h_{\lambda}=0.08$ (The solution of the system (1.3) and coefficients $b_{1}, b_{2}, \cdots, b_{10}$ for $h_{\lambda}=0.08$ are given in [2]) the wave parameters will be:

$y(0) \approx 14.8 \mathrm{~m} ; \quad y(\pi) \approx-6.9 \mathrm{~m} ; \quad H \approx 21.7 \mathrm{~m}$; $x_{c}=0, y_{c} \approx 0.4 \mathrm{~m} ; \quad T_{w} \approx 3.6 \times 10^{6} \mathrm{~kJ} ; \quad \Pi_{w} \approx 8.9 \times 10^{6} \mathrm{~kJ}$; $c \approx 28.1 \mathrm{~m} / \mathrm{s}$. This wave is unstable as $y_{c}(0.08)>0$.

In summary it is necessary to note that in the accessible publications we have not discovered materials for comparison, except [6].

\section{Conclusion}

Solving the Nekrasov's integral equation we avoided the "Liapunov-Schmidt" method and other methods of searching the solution in the neighbourhood of eigenvalues of Nekrasov's linearized equation. We sought the solution of this equation in the neighbourhood of the motionless point of the nonlinear integral Equation (1.5). This is the point $a_{1}^{0}=0.0474521226882492$;

$\mu_{1}^{0}\left(h_{\lambda}\right)=3.14159265358983 \operatorname{coth}\left(2 \pi h_{\lambda}\right)$. 


\section{REFERENCES}

[1] T. A. Bodnar', "One Approximate Solution of the Nekrasov Problem," Journal of Applied Mechanics and Technical Physics, Vol. 48, No. 6, 2007, pp. 818-823. doi:10.1007/s10808-007-0105-9

[2] T. A. Bodnar', "On Steady Periodic Waves on the Surface of a Fluid of Finite Depth," Journal of Applied Mechanics and Technical Physics, Vol. 52, No. 3, 2011, pp. 378-384. doi:10.1134/S0021894411030072

[3] T. A. Bodnar', "On Steady Waves on the Surface of a Finite-Depth Fluid," Free Boundary Problems: Theory, Experiment, and Applications, 3rd All-Russian Conference with International Participation, Biisk, 28 June-3 July 2008, pp. 25-26.

[4] A. I. Nekrasov, "Exact Theory of Steady Waves on the Surface of a Heavy Fluid," Izd.Akad.Nauk SSSR, Moskow, 1951. (In Russian)
[5] R. Courant and D. Hilbert, "Methods of Mathematical Physics," Interscience, New York, 1953.

[6] G. A. Chandler and I. G. Graham, "The Computation of Water Waves Modelled by Nekrasov's Equation," SIAM Journal on Numerical Analysis, Vol. 30, 1993, pp. 10411065. doi: $10.1137 / 0730054$

[7] R. Courant, "Differential and Integral Calculus," Interscience, New York, 1936.

[8] L. N. Sretenskii, "Theory of Fluid Wave Motion," Nauka, Moscow, 1977. (In Russian)

[9] T. A. Bodnar', "Conservation Law of the Full Mechanical Energy and Stability of the Steady-State Waves on the Surface of a Fluid of Finite Depth," In: IV All-Russian Conference with foreign participation on Free Boundary Problems: Theory, Experiment, and Applications, Biisk, 5-10 July 2011, pp. 18-19. 